Bull. Egypt. Soc. Physiol. Sci. Vol. (41), Issue (4), 470-483

\author{
Bull. of Egyp. Soc. Physiol. Sci. \\ (Official Journal of Egyptian Society for Physiological Sciences) \\ (pISSN: 1110-0842; eISSN: 2356-9514)
}

\title{
Ameliorative effect of sitagliptin and its cardinal mechanisms in methionine induced- hepatotoxicity in rats
}

\author{
Eman I. Elgizawy ${ }^{1}$, Amira A. Fouda ${ }^{2}$, Heba Fathy El domiaty ${ }^{1}$ \\ ${ }^{1}$ Clinical Physiology Department, Faculty of Medicine, Menoufia University, Menoufia, Egypt \\ ${ }^{2}$ Clinical Pathology Department, Faculty of Medicine, Menoufia University, Menoufia, Egypt
}

Submit Date: Dec 9, 2020

Revise Date: March 28, 2021

Accept Date: April 14, 2021

\section{Keywords}

- NALFD

- Methionine

- Sitagliptin

- Iron

- $\quad$ Reduced glutathione

\begin{abstract}
Background/ aim: High methionine (Met) (a precursor for homocysteine) diet is a risk factor for non-alcoholic fatty liver disease (NAFLD). This work demonstrated the hepatic effects of feeding western diet enriched with Methionine. Additionally, we evaluated the anti-oxidative properties of sitagliptin (STG) (an antidiabetic drug) which, counteract the negative effects of high Met diet. Methodology: Forty adult male Wister albino rats, divided into 4 group (10 rats/group) normal diet (control and STG groups), or high Methionine enriched diet, 1.5 \% Methionine (Met and Met + STG treated groups) for 35 days. Rats were either treated with vehicle (control, Met groups) or Sitagliptin, $100 \mathrm{mg} / \mathrm{kg} /$ day (STG, Met + STG groups). Investigations were Lipid profile, liver functions test, serum homocysteine, iron, ferritin, liver reduced glutathione (GSH), serum MDA level and histopathological investigations. Results: Met resulted in significant increase in LDL and cholesterol with significant decrease in HDL. Moreover, it had resulted in significant increase ALT and AST, with significant increase serum iron and homocysteine with no effect on serum ferritin, and significant decrease in tissue reduced glutathione as an antioxidant enzyme. STG with normal control diet had positive effects on different parameters. Treatment with STG has resulted in an improvement in most of altered parameters. Conclusions: Our findings suggest that Met induced NAFLD could be related to increase serum iron, homocysteine levels as an inflammatory activator factors and antioxidant machinery defects and the ameliorating role of STG in this type of induced hepatotoxicity.
\end{abstract}

Corresponding author: Eman Elgizawy Physiology Department, College of Medicine and Medical Science, Faculty of Medicine, Menoufia University, Menoufia, Egypt+201002947249, E-mail: eman.i.elgizawy@gmail.com 


\section{INTRODUCTION}

Non-alcoholic fatty liver disease (NAFLD) is believed to be major chronic liver injury locally and the most common liver disease universally [1]. It is characterized by accumulation of lipid in the absence of consumption of alcohol that progress to steatohepatitis, liver fibrosis, and eventually hepatocellular carcinoma (HCC) [2]. The leading hypothesis of disease progression is inspected to the increased susceptibility to inflammatory cytokines and oxidative stress [3]. Although many studies have been done on NAFLD, there are still numerous gaps related to our understanding of the underlying mechanisms.

NAFLD is generally presented asymptomatically and commonly companioning metabolic syndrome, obesity, type 2 diabetes mellitus (T2DM) and liver tissues pathological alterations [4]. NAFLD is fundamentally characterized by triacylglycerol accumulation in the liver tissue [5]. Ingestion of Western diet is a key inducer of fat accumulation in the liver, leading to dyslipidemia, insulin resistance (IR) and NAFLD [6,7]. Current treatments for NAFLD include lifestyle change (weight reduction), insulin sensitizer agents, lipid-lowering drugs and antioxidants[8]. Anti-diabetic drugs, which improve IR, have an outstanding effect on NAFLD and decelerate the pathology progression $[9,10]$.

Methionine is an essential amino acid that is metabolized by the liver mainly, where it is converted to S-adenosyl Methionine (SAMe). Although all mammalian cells synthesize SAMe, the liver is main site of formation SAMe as it is the organ where metabolism of about $50 \%$ of all dietary Methionine occurs. SAMe is mainly needed for methylation of a large variety of small molecules; So, if the concentration of SAMe elevated above certain level, the physiological hepatic functions will be also affected. There are many physiological conditions that affect the hepatic content of SAMe. In consequence, the control of these fluctuations requires the rate at which the liver synthesizes and catabolizes SAMe to be securely regulated. Therefore, maintaining SAMe homeostasis may be a therapeutic target in non-alcoholic steatohepatitis, alcoholic- and nonalcoholic liver cirrhosis [11].

Methionine is the precursor of homocysteine (Hcy), plasma sulphur amino acid homocysteine concentration is pointed out as a risk factor for vascular disease [12]. This hypothesis is of great interest because of the possibility that lowering blood homocysteine through nutritional interventions might prove to be safe and effective methods of reducing the disease associated risks.

Sitagliptin (STG), a recently developed dipeptidyl peptidase 4 inhibitor (DPP-4), which has been widely used to treat T2DM and has also been evaluated in diabetic patients with NAFLD symptoms [13]. So, the aim of present study is to investigate the effect of sitagliptin and its cardinal mechanisms in methionine induced- hepatotoxicity in rats.

\section{MATERIALS \& METHODS}

Ethics statement: The experimental protocol was approved by the local ethics committee of Faculty of Medicine, Menoufia University. Animals were housed (Medical Experimental Research lab, Faculty of Medicine, Menoufia University ,Egypt ) Animals were treated in accordance with Guide for the Care and Use of Laboratory Animals (eighth edition, National Academies Press) (NIH Publication No 85-23, Revised 1996) 
Animals: 40 adults male Wistar albino rats, matched for weight and age (between 150- 200 gm) were recruited for the study. Rats were purchased from a local providing facility. Rats were left to be acclimatized for 10 days. During the study, each of the 2 rats was kept in one cage at room temperature on standard rat chow and fresh water.

\section{Experimental design.}

Following acclimatization, animals were randomly allocated into four main groups (10 rats each):1. Control group: fed control diet which is normal rodent diet with $0.5 \%$ cholic acid and $2 \%$ maltose dextrin and gavaged with vehicle (water) for 35 days [14].

2. methionine (Met)- treated group: high Methionine diet was made by enriching the control diet with $1.5 \%$ Methionine (Sigma-Aldrich) and gavaged with vehicle (water) for 35 days $[15,16]$. 3. Sitagliptin (STG)- treated group: fed control diet and gavaged with an aqueous solution of Sitagliptin (Sigma-Aldrich) $100 \mathrm{mg} / \mathrm{kg} /$ day orally for $\quad 35$ days [14]. 4. Met + STG treated group: high Methionine diet was made by enriching the Control diet with $1.5 \%$ Methionine for 35 days and treated by gavege with an aqueous solution of Sitagliptin. At the end of the experiment (after 35 days), animals were fasted overnight, and blood samples were collected via cardiac puncture after rats being anaesthetized using $\mathrm{Na}^{+}$thiopental $(12 \mathrm{mg} / \mathrm{Kg}$ i.p.). Blood samples were left for clotting for 10 min and centrifuged at $4000 \mathrm{rpm}$ for another 10 min to isolate the serum and kept at $-20^{\circ} \mathrm{C}$ for further analysis of lipid profile, liver function test, homocysteine, iron and serum ferritin. Lastly, rats of all groups were sacrificed by cervical decapitation and the livers were excised and divided into two halves. One half was used for estimation of reduced GSH. The other half was used for histopathological and immunohistochemical investigations.

\section{Serum Biochemical Analysis}

\section{Lipid profile}

Total cholesterol (mg/dl) and HDL-C (mg/dl) levels were determined following their hydrolysis and oxidation to yield colored quinonimine derivatives using test reagent kits (Biodianostics, Egypt). Triglycerides (TGs) level (mg/dl) was estimated by a reagent kit (EMAPOL, Poland), in which TGs were hydrolyzed with lipoprotein lipase to form glycerol, which forms a complex with $\mathrm{H} 2 \mathrm{O} 2$ giving a colored derivative. The obtained levels of total cholesterol, HDL and TGs were then used to calculate the serum level of LDL-C as that described by Friedewald et al. [17].

$\mathrm{LDL}=$ Total Cholesterol - $(\mathrm{HDL}+$ Triglycerides/5).

\section{Assessment of Liver Function}

Blood was left to stand for $3 \mathrm{~h}$ in a centrifuge tube without anti-coagulant before the samples were centrifuged at $3000 \mathrm{rpm}$ at $4{ }^{\circ} \mathrm{C}$ for $10 \mathrm{~min}$ to separate the serum. For the liver function test, liver aspartate aminotransferase (AST) and alanine aminotransferase (ALT) activities were evaluated using the method by Reitman \& Frankel [18]. Serum and liver total protein were analyzed according to the method suggested by Bradford [19]. 


\section{Determination of serum iron and ferritin.}

Additional blood was collected into tubes with clot activator and gel separator. Cobas 8000 c701 (Roche) analyzer was used to determine iron concentration and unsaturated iron-binding capacity (UIBC). Transferrin saturation (TSAT) was calculated using formula: TSAT $(\%)=($ Serum Iron/Total Iron Binding Capacity) $\times 100 \%$. Total iron binding capacity (TIBC) was calculated as the sum of serum iron and UIBC values. Concentrations of serum ferritin and soluble transferrin receptor were measured using commercially available Elisa assays (Ferritin Rat Elisa Kit, Abnova and sTfR Immunoassay, R\&D Systems) according to the manufacturer's instructions [20].

\section{Determination of serum homocysteine (Hcy)}

Serum total Hcy concentrations were determined by HPLC (high performance liquid chromatography) equipped with a fluorometric detection system [21].

\section{Assay of hepatic reduced (GSH) concentrations}

To obtain a $10 \%(\mathrm{w} / \mathrm{v})$ liver homogenate, $1 \mathrm{~g}$ of liver tissue was homogenized with $9 \mathrm{~mL}$ of $1.15 \%$ $\mathrm{KCl}$. The liver homogenate was then centrifuged at $10,000 \times g$ for $15 \mathrm{~min}$ at $4{ }^{\circ} \mathrm{C}$. The resulting supernatant was used to determine the contents of reduced GSH and lipid peroxides as well as the activities of GSH-related enzymes. The GSH content in the liver homogenate was determined by high performance liquid chromatography (HPLC)/mass spectrometer (MS) [22].

\section{Determination of Malondialdehyde Serum Levels}

Malondialdehyde was extracted from serum samples by treatment with 20 percent TCA at ratio of $1: 1$. The serum samples were centrifuge at 8000 $\mathrm{rpm}$ and filtered through a $0.2 \mu \mathrm{m}$ membrane. The supernatant was treated Thiobarbituric acid (20 $\mathrm{mM}$ ) and boiled in a water bath for $60 \mathrm{~min}$. A 10 $\mu \mathrm{L}$ sample was injected into a high-performance liquid chromatography (HPLC) (Merck Hitachi) and separated with a C-18 phanomenex column, model RP-18, and detected with an HPLC fluorescence detector (Shimadzu, Japan) set at 532 $\mathrm{nm}$ excitation and $553 \mathrm{~nm}$ emission. The mobile phase consisted of a 35:65 $(v / v)$ mixture of methanol and $0.05 \mathrm{M}$ potassium phosphate buffer, $\mathrm{pH} \mathrm{7}$, and the flow rate was $1 \mathrm{~mL} / \mathrm{min}$. MDA standard solutions were used to generate a standard curve. The nM levels of MDA found by us in the tested cohort are in agreement with serum MDA levels previously found in human after several hours of fasting[23]. High MDA levels were defined above the sample median corresponding to 12.87, 12.10, and $13.46(\mathrm{nM})$ among the entire sample, men and women, respectively.

\section{Histopathological examination}

Liver tissues were fixed in $10 \%$ formalin, embedded in paraffin and sectioned at $5 \mu \mathrm{m}$ thickness. Changes in liver pathology and collagen deposition were observed by hematoxylin-eosin $(\mathrm{H}$ and E) staining [24].

\section{Statistical Analysis}

Results are expressed as mean \pm standard error (SE). Kolmogorov-Smirnov test was performed on all data sets to ensure normal distribution $(p>0.5)$. Student t-test or repeated-measures Analysis of Variances (ANOVA) were used for statistical analysis of the different groups which ever appropriate, using Origin ${ }^{\circledR}$ software and the probability of chance ( $p$ values). $\mathrm{P}$ values $<0.05$ were considered significant. 


\section{RESULTS}

Serum AST, ALT levels increased significantly in the Met group when compared to the control group $(73.6 \pm 1.4 \mathrm{vs} 41.5 \pm 0.9 \mathrm{mg} / \mathrm{dl})(65.9 \pm 0.9$ vs $31.4 \pm 1.03 \mathrm{mg} / \mathrm{dl}, \mathrm{P}<0.05)$. Their levels were significantly lower in the Met + STG treated group than that in the Met group $(46.1 \pm 2 \mathrm{mg} / \mathrm{dl}$ vs 73.6 $\pm 1.4, \mathrm{P}<0.05)(35.4 \pm 1.9 \mathrm{mg} / \mathrm{dl}$ vs $65.9 \pm 0.9, \mathrm{P}$ $<0.05)$. There was no statistically significant difference in serum AST between STG treated, Met + STG and control groups $(43.8 \pm 0 . \mathrm{mg} / \mathrm{dl}$ vs $46.1 \pm 2$ and $41.5 \pm 0.9 \mathrm{mg} / \mathrm{dl}$, respectively, $\mathrm{P}>$ 0.05). There was no statistically significant difference in serum ALT between Met + STG and control groups $(35.4 \pm 1.9$ vs $31.4 \pm 1.03, \mathrm{P}>$ 0.05), however serum ALT levels in Met + STG treated group were significantly higher compared to STG treated group $(35.4 \pm 1.9$ vs $25.5 \pm 1.3, \mathrm{P}<$ 0.05) (Fig.1.a).

Serum cholesterol levels were significantly higher in the Met group when compared to the control group $(263.8 \pm 2.7$ vs $168.6 \pm 2.2 \mathrm{mg} / \mathrm{dl}, \mathrm{P}$ $<$ 0.05). Serum cholesterol levels were significantly lower in Met + STG treated group when compared to the Met group (177.6 \pm $2.8 \mathrm{mg} / \mathrm{dl} \mathrm{P}<0.05$ ), and insignificant to corresponding value in the control group, while serum cholesterol levels were significantly higher in Met + STG treated group compared to STG treated group (123.9 $\pm 3.1, \mathrm{P}<0.05)$ (Fig. 1.b).

Serum TG level was insignificantly different in control, Met, STG and Met + STG groups $(66.5 \pm 2.6,65.7 \pm 1.8,70.9 \pm 1.5$ and $75 \pm 1.3$ $\mathrm{mg} / \mathrm{dl}$, respectively ( $\mathrm{P}>0.05$ (Fig. 1.b).

Serum HDL level decreased significantly in the Met group when compared to the control group $(18.7 \pm 0.8$ vs $41.6 \pm 0.6 \mathrm{mg} / \mathrm{dl}, \mathrm{P}<0.05)$. it was significantly higher in the Met + STG treated group than that in the Met group $(37.4 \pm 2 \mathrm{mg} / \mathrm{dl}, \mathrm{P}<$ 0.05). There was no statistically significant difference between Met +STG and control groups, while it was still significantly lower in Met +STG treated group compared to STG treated group (50.5 $\pm 2.2 \mathrm{P}<0.05)$ (Fig.1. b).

Serum LDL levels were significantly higher in the Met group when compared to the control group $(231.6 \pm 3.2$ vs $113.6 \pm 2.2, \mathrm{P}<0.05)$. It was significantly lower in Met + STG group when compared to the Met group $(119.6 \pm 3.3 \mathrm{mg} / \mathrm{dl}, \mathrm{P}<$ 0.05). Fortunately, there was insignificant difference between Met+ STG group and control group, while It was significantly higher in Met +STG treated group compared to STG treated group (59.2 $\pm 2.4, \mathrm{P}<0.05)$ (Fig. $\underline{1 . b})$.

Serum iron level was significantly higher in the Met group when compared to the control group $(0.35 \pm 0.009$ vs $0.22 \pm 0.009 \mathrm{mg} / \mathrm{dl}, \mathrm{P}<0.001)$. Serum iron level was significantly lower in Met + STG treated group when compared to the Met group $(0.2 \pm 0.018 \mathrm{mg} / \mathrm{dl}, \quad \mathrm{P}<0.001)$, and insignificantly different, compared to the corresponding value in the control group, while it was significantly lower to the corresponding value in STG treated group $(0.14 \pm 0.004 \mathrm{mg} / \mathrm{dl}, \mathrm{P}<$ 0.05) (Fig.2.a).

Serum ferritin level was insignificantly different in control, Met, STG and Met + STG groups $(118.5 \pm 1.8,117.8 \pm 1.04,118 \pm 2.6$ and $118 \pm 2.4$ ng/dl, respectively ( $\mathrm{P}>0.05)$ (Fig. 2.b).

Serum homocysteine level was significantly higher in the Met group when compared to the control group $(24.8 \pm 1$ vs $14.2 \pm 0.6 \mathrm{Mmol} / 1, \mathrm{P}<$ $0.05)$. It was significantly lower in Met $+\mathrm{STG}$ treated group when compared to the Met group 
$(13.8 \pm 0.7$ vs $24.8 \pm 1 \mathrm{Mmol} / \mathrm{l}, \mathrm{P}<0.05)$, and insignificantly in Met+ STG to corresponding value in the control group, STG group (13.8 \pm $0.7 \mathrm{mg} / \mathrm{dl}$ vs $14.2 \pm 0.6$ and $13.5 \pm 0.8 \mathrm{Mmol} / \mathrm{l}$, respectively) $(\mathrm{P}>0.05)$ (Fig. 2.c).

Liver reduced glutathione level was significantly lower in the Met group when compared to the control group $(0.5 \pm 0.4$ vs $1.5 \pm$ $0.06 \mathrm{mmol} / \mathrm{mg}, \quad \mathrm{P}<0.05)$. Liver reduced glutathione was significantly lower in Met + STG treated aged group $(1.5 \pm 0.12 \mathrm{mmol} / \mathrm{mg}, \mathrm{P}<$ 0.005) when compared to the Met group, and insignificantly to corresponding value in the control group, STG group $(1.7 \pm 0.5 \mathrm{mmol} / \mathrm{mg}, \mathrm{P}>$ 0.05) (Fig. 3.a)

Serum MDA level was significantly higher in the Met group when compared to the control group $(157.7 \pm 5.5$ vs $67.3 \pm 2.4 \mathrm{nmol} / \mathrm{ml}, \mathrm{P}<0.05)$. It was significantly higher in Met + STG treated aged group $(75.1 \pm 3.1 \mathrm{P}<0.05)$ when compared to the Met group, and insignificantly to corresponding value in the control group, STG group (68.4 $\pm 5.3, \mathrm{P}>0.05)$ (Fig. 3.b)

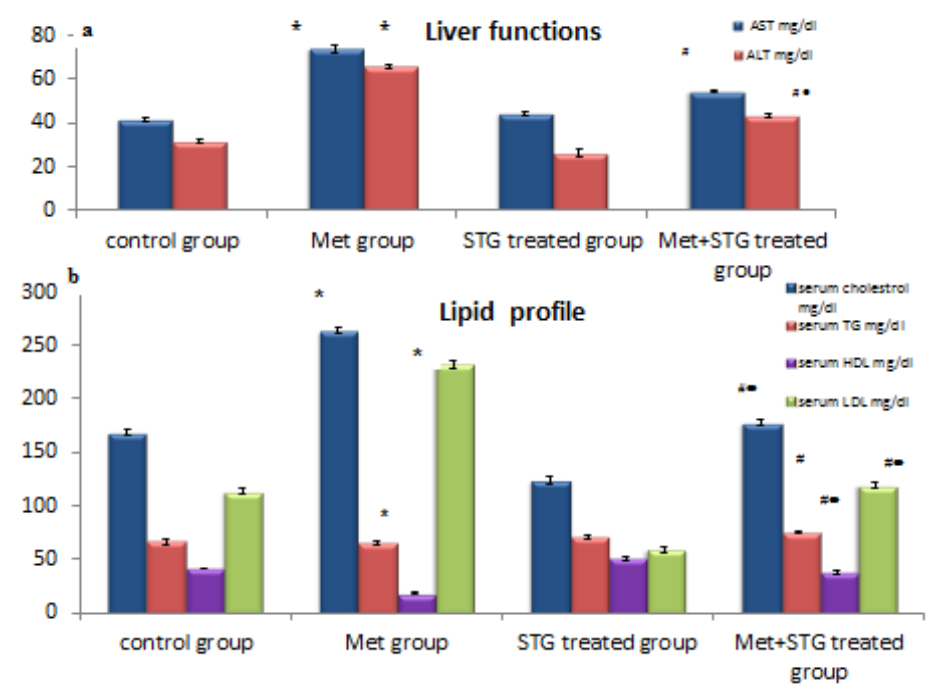

Fig (1): STG attenuates Met-induced hepatotoxic effect. a. Effect of STG treatment on serum level of AST and ALT in control, Met, STG and Met+ STG treated rats b. Effect of STG treatment on serum level of cholesterol, TG, HDL and LDL in control, Met, STG and Met + STG treated rats.

* Significant when compared to control group, \# Significant, when compared to Met group, • Significant, when compared to STG treated group $(n=10)$.

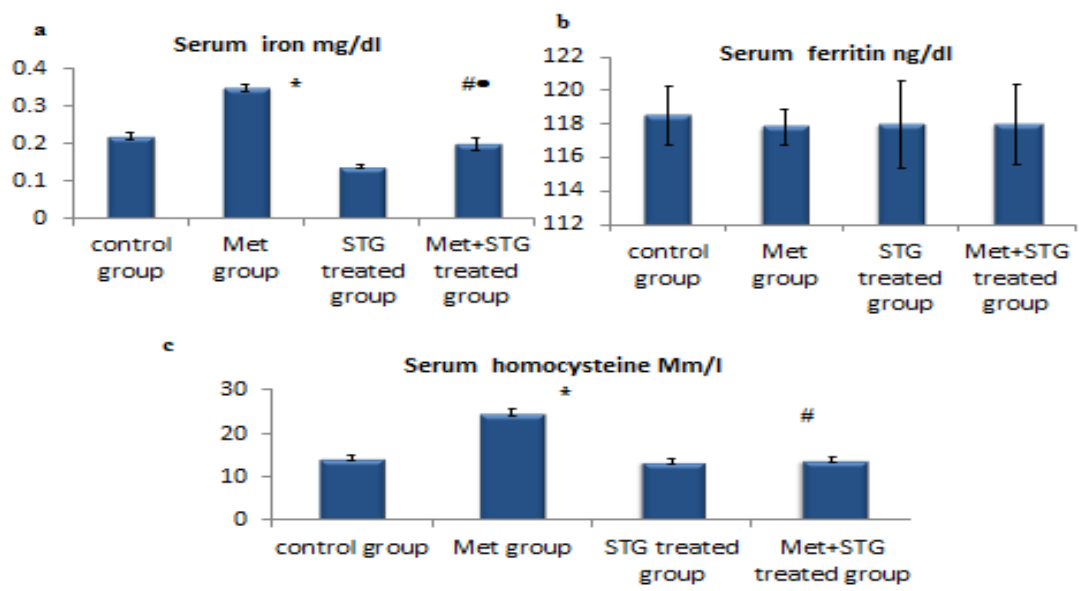

Fig (2): a. Effect of STG treatment on level of serum iron in control, Met, STG and Met+ STG treated rats. b. Effect of STG treatment on level of serum ferritin in control, Met, STG and Met+ STG treated rats c. Effect of STG treatment on level of serum homocysteine in control, Met, STG and Met+ STG treated rats.

*Significant when compared to control group, \# Significant when compared to Met group. $\bullet$ Significant when compared to STG treated group $(n=10)$. 


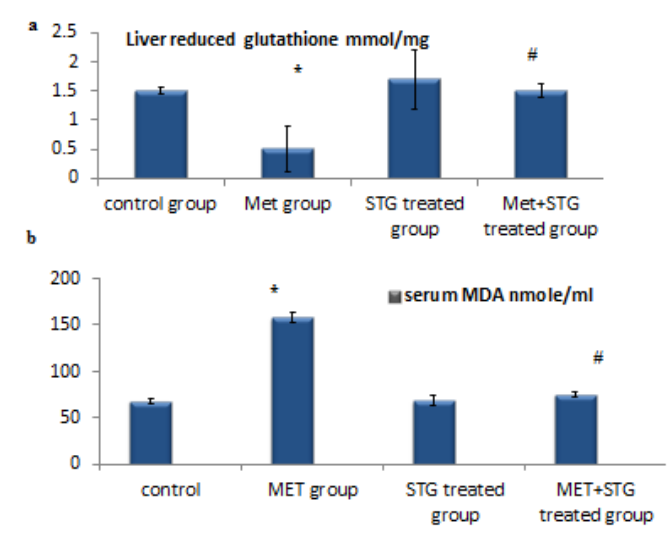

Fig (3): STG attenuates Met-inflammatory and oxidant effect. a. Effect of STG treatment on liver reduced glutathione in control, Met, STG and Met + STG treated rats. b. Effect of STG treatment on serum level of MDA in control, Met, STG and Met + STG treated rats

Fig (4): histopathological examination of rat liver showing:

a.

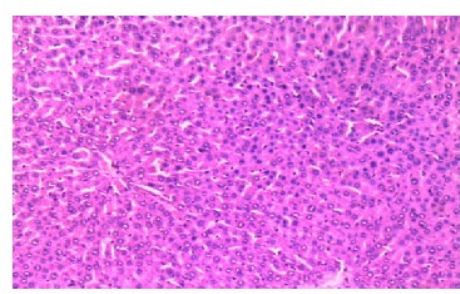

.c.

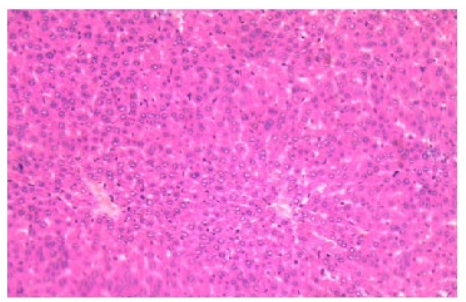

b.

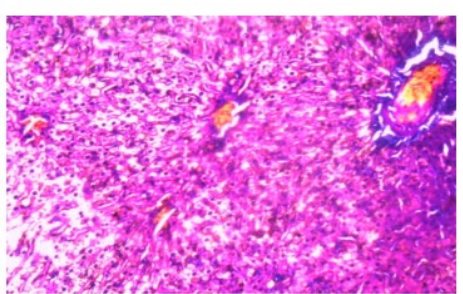

d.

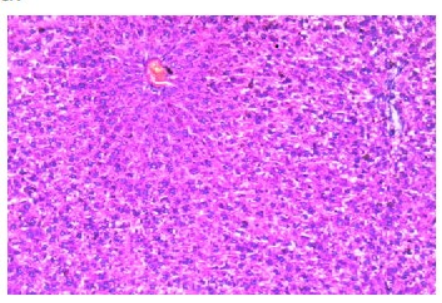

Fig (4): a. control group showed normal liver showing hexagonal structure of normal hepatocytes with intervening portal tracts (H\&E X 100), b. Met group showed Fatty liver disease showing signet ring appearance of the liver cells, fibrosis and inflammatory infiltrate at the portal tract, (H\&E X 100) c. STG group showed no significant changes in liver tissue showing hepatic lobules formed of cords of hepatocytes (H\&Ex100), d. Met + STG showed Liver restored its normal architecture after sitagliptin treatment (H\&E X 100).

\section{DISSCUSSION.}

NAFLD is considered the most common cause of liver diseases especially in developed countries [25]. There is a common association between NAFLD, type 2 DM and metabolic syndrome [26]. NAFLD is characterized by hepatic cell damage, inflammation, and liver fibrosis which may progress to cirrhosis [27]. Most Western diets are composed of animal products like meat, dairy and poultry, all of these are rich in Methionine. Although Methionine has been independently associated with NAFLD, the exact mechanism by which Methionine leads to NAFLD is not completely studied.

Enzyme dipeptidyl peptidase 4 (DPP-4) is widely distributed in many organs throughout the body including liver. There are many evidences suggest that DPP-4 is involved in the pathogenesis of many chronic liver diseases such as hepatitis $\mathrm{C}$ virus (HCV) infection, NAFLD, and hepatocellular carcinoma. Sitagliptin, is an oral hypoglycemic agent that act as DPP-4 competitive inhibitor, 
inactivates glucagon-like peptide-1 (GLP-1) hormone and glucose-dependent insulinotropic polypeptide (GIP) released in relation to meals [28]. So, it inhibits hyperglycemia and hyperinsulinemia and improves fatty liver [29]. But there is no study examined the effect of Sitagliptin on fatty liver in absence of diabetes. So, the aim of the present study was to investigate the effect of Sitagliptin on Methionine induced fatty liver in non-diabetic albino rats.

As shown in the current results, high Methionine enriched diet increased liver enzymes, lipid profiles, serum iron, and serum homocysteine and decreased tissue reduced glutathione. While treatment by Sitagliptin decreased liver enzymes, lipid profiles, serum iron, serum homocysteine and increased tissue reduced glutathione.

The hepatic damaging effect of high Methionine diet in the current study was in agreement Gomez et al. (2009), who explained it by increased mitochondrial reactive oxygen species (ROS) production and the oxidative damage in rat liver mitochondrial DNA [30]. Additionally, the study by Yamada and coworkers demonstrated that feeding high Methionine diet induces liver cells injury in mice via changing the hepatic lipid metabolism and inducing oxidative stress [31].

As demonstrated in this study, there was significant increase in serum homocysteine in fatty liver group compared to control group. This can be explained by the fact that, the $S$ adenosylmethionine (SAM), the activated form of Methionine, is converted to Sadenosylhomocysteine (SAH) after donating its methyl group to methyl group acceptor molecules. Then, it is de-adenosylated to homocysteine. After intake of high Methionine diet, this metabolic end product of the trans-methylation cycle of Methionine metabolism increases resulting in hyperhomocysteinemia (HHcy). It has been demonstrated that excess SAM, is a leading cause for hepatic steatosis and the development of NAFLD [32]. Furthermore, it was reported that hyperhomocysteinemia triggers hepatic steatosis by activating endoplasmic reticulum (ER) stress responses [33]. This ER stress responses lead to producing oxidative stress, inflammatory cell infiltration and fibrosis [34]. Moreover, high levels of Hcy thiolactone (HTL); an intramolecular thioester of Hcy, as a result of hyperhomocysteinemia were demonstrated in many human and animal studies [35]. Interestingly, HTL has been shown to induce oxidative stress [36] which has been involved in many pathological conditions associated with hyperhomocysteinemia [37]. Previous studies had successfully reported the pathophysiological outcome of hyperhomocysteinemia in vivo by using a high-Methionine/low-folate diet or genemodified animal models [38].

Furthermore, in the same line with the results of the current study, it has been demonstrated that diet supplemented with Methionine increased plasma hydroperoxides and LDL-cholesterol [39], serum cholesterol due to increased hepatic cholesterol synthesis [40], raised iron and lipid peroxidation, conjugated dienes in rat liver, alters liver antioxidant enzymes and glutathione [41].

Sitagliptin is an oral anti-diabetic drug, has been proven to have antioxidant and anti-inflammatory effects in many body organs $[42,43,44]$. Regardless the hypoglycemic effect of Sitagliptin, it has been documented to have other beneficial effects in diabetic patients. Among them, it 
ameliorates liver steatosis by inhibiting fatty acid synthesis in the liver [45,46]. Additionally, Sitagliptin was shown to improve the oxidative stress responses in diabetic nephropathy [47]. On the opposite side, there is only one study by Kumar et al. [14], stated that sitagliptin increased hepatic markers of oxidative stress in rats fed a high Cholesterol diet. But none of these studies have investigated the possibility of the hepatic protective effect of Sitagliptin after feeding with high Methionine diet only.

In the study performed by Onovama [48], it has been reported that treatment with Sitagliptin enhanced peroxisome proliferator activated receptor (PPAR- $\alpha$ ) and attenuated sterol regulatory element-binding protein 1c (SREBP1c) and (fatty acid synthase) FAS. In other words, Sitagliptin decreased hepatic steatosis by attenuating lipogenesis and stimulating lipolysis. Also, it has been shown that DPP4i enhances the hepatic levels of phosphorylated AMP-activated protein kinase, which leads to suppression of de novo lipogenesis. Therefore, our results are in agreement with those of previous studies, and the effect of DPP4i on hepatic lipid metabolism may be the mechanism for improving steatohepatitis.

There is great evidence that excess iron is usually associated with more liver injury in NAFLD, although the exact mechanisms are still unclear [49]. The effect of hepatic iron on the pathogenesis of NAFLD is usually due to production of reactive oxygen species by iron. In non- alcoholic steatohepatitis (NASH), oxidative stress causes cell death through depletion of ATP, NAD and glutathione, and by direct DNA, lipids and proteins damage inside liver cells.
Furthermore, oxidative stress leads to an increase in the production of pro-inflammatory cytokines and a fibro genic response [50]. Therefore, the ability of Sitagliptin to reduce serum iron level, as demonstrated in the current results can be used as an explanation for the associated increase in the hepatic reduced glutathione level, as an antioxidant parameter, and the associated improvement in liver functions parameters.

It is well known that homocysteine is correlated with oxidative stress formation [51]. There are many studies found a significant association between NASH and higher level of HCY [52]. Moreover, some studies found negative correlation between HCY values and reduced glutathione (GSH) [53,54]. This suggests the real role of hyperhomocystinemia in the pathogenesis of NAFLD. In this study, Sitagliptin succeed to reduce serum homocysteine levels and so protect liver cells from the damaging effect of homocysteine. This was in agreement with the studies who reported that Linagliptin, Sitagliptin, and MK-0626 can improve hepatic steatosis $[55,56,57]$. Additionally, there was a clinical study which demonstrated that Vildagliptin can decrease liver triglyceride content by about $27 \%$ in type 2 diabetic patients [58]. Moreover, Vildagliptin was found to decrease ER stress. ER stress occurs when protein maturation in the ER is impaired, which leads to a characteristic stress response named the unfolded protein response (UPR). However, prolonged activation of UPR is correlated with many metabolic diseases, such as insulin resistance, type 2- diabetes, and hepatic steatosis [59]. So, when ER stress is inhibited hepatic steatosis will be decreased [60]. 
The results of Wang et al. (2018) have reported that treatment of NASH and liver fibrosis with Sitagliptin and Linagliptin significantly decreased parameters of steatosis and inflammation. Gliptins, via suppression of inflammation decrease steatosis, apoptosis, oxidative stress, and vascular dysfunction in murine models of NASH and liver fibrosis, with mild direct anti-fibrotic properties. They reduce the numbers of liver and vascular inflammatory monocytes/macrophages and induce their alternative polarization, with beneficial effect on NASH-associated hepatic fibrosis [61]. Unfortunately, Joy and his coworkers found that Sitagliptin did not improve the fibrotic score or NASH after 24 weeks of therapy. This may be due to the different dose and duration of therapy [62].

\section{Conclusion:}

The present study elucidates that sitagliptin as a DDP4i can improve NAFLD, which was induced by high methionine diet and preserves the liver function. The study revealed that oxidative stress, increase serum iron and serum homocysteine could be some of the underlying mechanisms in methionine induced hepatotoxicity.

\section{Conflict of interest:}

The authors elucidate that there is no conflict of interest associated with this work.

\section{Funding.}

This research did not receive any specific grant from funding agencies in the public, commercial, or not-forprofit sectors

\section{Acknowledgement.}

The authors thank the technicians of Central Lab of Faculty of Medicine, Menoufia University, Egypt for their great help in performing the biochemical assays.

\section{References}

1. Loomba R, Sanyal AJ. The global NAFLD epidemic. Nat Rev Gastroenterol Hepatol. 10(11):686-690; 2013.

\section{Chalasani N, Younossi Z, Lavine JE, Diehl} AM, Brunt EM, Cusi K, Charlton M, Sanyal AJ, American Gastroenterological Association. American Association for the Study of Liver Diseases. American College of Gastroenterologyh .The diagnosis and management of non-alcoholic fatty liver disease: practice guideline by the American Gastroenterological Association, American Association for the Study of Liver Diseases, and American College of Gastroenterology. Gastroenterology.;142(7):15921609. 2012.

\section{Browning JD, Horton JD. Molecular} mediators of hepatic steatosis and liver injury. $J$ Clin Invest;114(2):147-152;2004.

4. Cohen JC, Horton JD, Hobbs HH. Human fatty liver disease: old questions and new insights. Science.;332(6037):1519-1523;2011.

5. Angulo P. Nonalcoholic fatty liver disease. $N$ Engl J Med.; 346:1221-1231; 2002.

6. Dowman JK, Tomlinson JW, Newsome PN. Pathogenesis of non-alcoholic fatty liver disease. QJM; 103:71-83;2010

\section{Hassan K, Bhalla V, El Regal ME, A-Kader} HH. Nonalcoholic fatty liver disease: A comprehensive review of a growing epidemic. World J Gastroenterol.20:12082-12101.;2014

\section{Fruci B, Giuliano S, Mazza A, Malaguarnera}

R, Belfiore A. Nonalcoholic Fatty liver: A possible new target for type 2 diabetes prevention and treatment. Int $J$ Mol Sci; 14:2293322966.;2013 
9. Leite NC, Villela-Nogueira CA, Cardoso CR, Salles GF. Non-alcoholic fatty liver disease and diabetes: From physiopathological interplay to diagnosis and treatment. World $J$ Gastroenterol; 20:8377-8392;2014

10. Higuera-de la Tijera F, Servín-Caamaño AI. Pathophysiological mechanisms involved in nonalcoholic steatohepatitis and novel potential therapeutic targets. World J Hepatol.; 7:12971301;2015

11. Mato J, Martínez-Chantar M, and Luz S. S-adenosyl Methionine metabolism and liver disease. Ann Hepatol; 12(2): 183-189;2013

12. Hui E, Xu A, Yang Bo H, Lam KS. Obesity as the common soil of non-alcoholic fatty liver disease and diabetes: Role of adipokines. J Diabetes Investig.; 4:413-425; 2013

13. Blaslov K, Bulum T, Zibar $K$ and Duvnjak L: Incretin based therapies: A novel treatment approach for non-alcoholic fatty liver disease. World J Gastroenterol 20: 7356-7365, 2014.

\section{Kumar A, Pathak R, Palfrey H, Stone K,} Gettys K and Subramanyam N. Murthy1 umar. High levels of dietary Methionine improve Sitagliptin-induced hepatotoxicity by attenuating oxidative stress in hypercholesterolemic rats. Nutrition \& Metabolism 17:2;2020

15. Yamada H, Akahoshi N, Kamata S, Hagiya Y, Hishiki T, Nagahata Y,MatsuuraT, Takano N, Mori M, Ishizaki Y. Methionine excess in diet induces acute lethal hepatitis in mice lacking cystathionine $\gamma$-lyase, an animal model of cystathioninuria. Free Radic Biol Med.;52(9):1716-26; 2012.

16. Dai H, Wang W, Tang $X$, Chen $R$, Chen $Z$, Lu Y, Yuan H. Association between homocysteine and non-alcoholic fatty liver disease in Chinese adults: a cross-sectional study. Nutr J.;15(1):102; 2016.

17. Friedewald WT, Levy RI and Fredrickson DS . Estimation of the concentration of lowdensity lipoprotein cholesterol in plasma, without use of the preparative ultracentrifuge. Clin Chem. 18(6):499-502;1972.

18. Reitman S, Frankel S. Colorimetric method for the determination of serum transaminase activity. Am J Clin Pathol.; 28:56-63.;1957.

19. Bradford MM. A rapid and sensitive method for the quantitation of microgram quantities of protein utilizing the principle of protein-dye binding. Anal Biochem; 72:248-254.;1976.

20. Paterek A, Kępska M, Sochanowicz B, Chajduk E, Kolodziejczyk J, PolkowskaMotrenko H, Kruszewski M, Leszek P, Mackiewicz U \& Mączewski M Beneficial effects of intravenous iron therapy in a rat model of heart failure with preserved systemic iron status but depleted intracellular cardiac stores. 8:15758;2018.

21. Araki A, Sako Y. Determination of free and total homocysteine in human plasma by highperformance liquid chromatography with fluorescence detection. J Chromatogr; 422: 43-52. 1987.

\section{Guan X, Hoffman B, Dwivedi, C\&} Matthees, D.P. A simultaneous liquid chromatography/mass spectrometric assay of glutathione, cysteine, homocysteine and their disulfides in biological samples. J. Pharm. Biomed. Anal., 31, 251-261;2003.

\section{Gorelik S., Ligumsky M., Kohen R., Kanner}

J. A novel function of red wine polyphenols in humans: Prevention of absorption of cytotoxic 
lipid peroxidation products. FASEB J.;22:4146.;2008.

24. Chevallier M, Guerret S, Chossegros P, Gerard F, Grimaud JA. A histological semiquantitative scoring system for evaluation of hepatic fibrosis in needle liver biopsy specimens: Comparison with morphometric studies. Hepatology.; 20:349-551994; 1994.

25. Anstee QM, Targher G, Day CP. Progression of NAFLD to diabetes mellitus, cardiovascular disease or cirrhosis. Nat Rev Gastroenterol Hepatol.; 10:330-344.;2013.

26. McPherson S, Hardy T, Henderson E, Burt AD, Day CP, Anstee QM. Evidence of NAFLD progression from steatosis to fibrosingsteatohepatitis using paired biopsies: implications for prognosis and clinical management. J Hepatol. 62:1148-1155;2015.

27. Adams LA, Sanderson S, Lindor KD, Angulo P. The histological course of nonalcoholic fatty liver disease: a longitudinal study of 103 patients with sequential liver biopsies. $J$ Hepatol. 42:132-138;2005.

28. Herman GA, Bergman A, Liu F, et al. Pharmacokinetics and pharmacodynamics effects of the oral DPP-4 inhibitor sitagliptin in middleaged obese subjects. J Clin Pharmacol.; 46:876886. 2006.

29. Ferre P, Foufelle F. Hepatic steatosis: a role for de novo lipogenesis and the transcription factor SREBP-1c. Diabetes Obes Metab.; 12:83-92; 2010.

30. Gomez, J., Caro, P., Sanchez, I., Naudi, A., Jove, M., Portero-Otin, M., ... \& Barja, G. (). Effect of methionine dietary supplementation on mitochondrial oxygen radical generation and oxidative DNA damage in rat liver and heart.
Journal of bioenergetics and biomembranes,: 41(3), 309-321. 2009.

\section{Yamada H, Akahoshi N, Kamata S, Hagiya} Y, Hishiki T, Nagahata Y, Matsuura T, Takano N, Mori M, Ishizaki Y. Methionine excess in diet induces acute lethal hepatitis in mice lacking cystathionine $\gamma$-lyase, an animal model of cystathioninuria. Free Radic Biol Med.;52(9):1716-26. 2012.

32. Martínez-Uña M, Varela-Rey M, Cano A, Fernández-Ares L, Beraza N, Aurrekoetxea I, Martínez-Arranz I, García-Rodríguez JL, Buqué $\mathrm{X}$, Mestre D. Excess Sadenosylmethionine reroutes phosphatidylethanolamine towards phosphatidylcholine and triglyceride synthesis. Hepatology.;58(4): 1296-305. 2013.

\section{Ai Y, Sun Z, Peng C, Liu L, Xiao X, Li J.} Homocysteine induces hepatic steatosis involving ER stress response in high methionine diet-fed mice. Nutrients.;9(4):346. 2017.

34. Matte C, Stefanello FM, Mackedanz V, Pederzolli CD, Lamers ML, Dutra-Filho CS, dos Santos MF, Wyse AT. Homocysteine induces oxidative stress, inflammatory infiltration, fibrosis and reduces glycogen/glycoprotein content in liver of rats. Int J Dev Neurosci.;27(4):337-44. 2009.

35. Jakubowski H. The pathophysiological hypothesis of homocysteine thiolactone-mediated vascular disease. J Physiol Pharmacol.;59(Suppl 9):155-67. 2008.

36. Yang XH, Li P, Yin YL, Tu JH, Dai W, Liu LY, Wang SX. Rosiglitazone via PPAR $\gamma$ dependent suppression of oxidative stress attenuates endothelial dysfunction in rats fed homocysteine thiolactone. $J$ Cell Mol Med.; 19(4):826-35. 2015 
37. Tyagi N, Sedoris KC, Steed M, Ovechkin AV, Moshal KS, Tyagi SC. Mechanisms of homocysteine-induced oxidative stress. Am J Phys Heart Circ Phys.;289(6):H2649-56; 2005.

38. Obeid, R.; Herrmann, W. Homocysteine and lipids: S-Adenosyl methionine as a key intermediate. FEBS Lett., 583, 1215-1225. 2009

39. Hidiroglou N, Gilani GS, Long L, Zhao X, Madere R, Cockell K, Belonge B, Ratnayake WMN, Peace R J. Nutr Biochem 15:730-740; 2004.

40. Hirche, F., Schröder, A., Knoth, B., Stangl, G. I., \& Eder, K. Effect of dietary methionine on plasma and liver cholesterol concentrations in rats and expression of hepatic genes involved in cholesterol metabolism. British journal of nutrition, 95(5), 879-888; 2006.

41. Mori N, Hirayama K J Nutr: 130:2349-2355; 2000 .

42. Fan M, Li Y, Zhang S. Effects of sitagliptin on lipid profiles in patients with type 2 diabetes mellitus: a meta-analysis of randomized clinical trials. Medicine (Baltimore). 2016;95(2):e2386. M, Li Y, Zhang S. Effects of sitagliptin on lipid profiles in patients with type 2 diabetes mellitus: a meta-analysis of randomized clinical trials. Medicine (Baltimore).;95(2):e2386;2016.

43. Alam MA, Chowdhury MRH, Jain P, Sagor MAT, Reza HM. DPP-4 inhibitor sitagliptin prevents inflammation and oxidative stress of heart and kidney in two kidney and one clip (2K1C) rats. Diabetol Metabol Syndr;7(1):107;2015.

44. Samson SL, Bajaj M. Potential of incretinbased therapies for non-alcoholic fatty liver disease. J Diabetes Complicat.;27(4):401-6;2013.

45. Sujishi T, Fukunishi S, Ii M, Nakamura K, Yokohama K, Ohama H, Tsuchimoto Y, Asai
A, Tsuda Y, Higuchi K. Sitagliptin can inhibit the development of hepatic steatosis in high-fructose diet-fed $\mathrm{Ob} / \mathrm{Ob}$ mice. $J$ Clin Biochem Nutr. 2015;57(3):244-53.

46. Shen T, Xu B, Lei T, Chen L, Zhang C, Ni Z. Sitagliptin reduces insulin resistance and improves rat liver steatosis via the SIRT1/AMPK $\alpha$ pathway. Exp Ther Med. 2018;16(4):3121-8.

47. Civantos E, Bosch E, Ramirez E, Zhenyukh O, Egido J, Lorenzo O, Mas S. Sitagliptin ameliorates oxidative stress in experimental diabetic nephropathy by diminishing the miR200a/Keap-1/Nrf2 antioxidant pathway. Diabetes Metab Syndr Obes. 2017; 10:207.

48. Onoyama T, Koda M, Okamoto T, Kishina M, Matono T, Sugihara $T$ and Murawaki $Y$ : Therapeutic effects of the dipeptidyl peptidase-IV inhibitor, sitagliptin, on non-alcoholic steatohepatitis in FLS-ob/ob male mice. Mol Med Rep 12: 6895-6902, 2015.

49. Nelson JE, Klintworth H, Kowdley KV. Iron metabolism in Nonalcoholic Fatty Liver Disease. Curr Gastroenterol Rep.; 14:8-16; 2012.

50. Rolo AP, Teodoro JS, Palmeira CM. Role of oxidative stress in the pathogenesis of nonalcoholic steatohepatitis. Free Radic Biol Med.; 52:59-69. 2012

51. Arguello G, Balboa E, Arrese M, Zanlungo S. Recent insights on the role of cholesterol in non-alcoholic fatty liver disease. Biochim Biophys Acta (BBA)-Mol Basis Dis.;1852(9):1765-78. 2015.

52. de Carvalho, S.C.R., Muniz, M.T.C., Siqueira, M.D.V. et al. Plasmatic higher levels of homocysteine in Non-alcoholic fatty liver disease (NAFLD). Nutr J 12, 37 ;2013. 
53. Leach NV, Dronca E, Vesa SC, et al. Serum homocysteine levels, oxidative stress and cardiovascular risk in non-alcoholic steatohepatitis. Eur J Intern Med. 25:762-67; 2014.

54. Pastore A, Alisi A, di Giovamberardino G, et al. Plasma levels of homocysteine and cysteine increased in pediatric NAFLD and strongly correlated with severity of liver damage. Int $\mathrm{J} \mathrm{Mol}$ Sci; 15:21202-14; 2014.

55. Kern M, Klöting $N$, Niessen $H$ et al., "Linagliptin improves insulin sensitivity and hepatic steatosis in diet-induced obesity," PLoS ONE, vol. 7, no. 6, Article ID e38744, 2012.

56. Ohyama T, Sato K, Yamazaki Y et al., "MK0626, a selective DPP-4 inhibitor, attenuates hepatic steatosis in ob/ob mice," World Journal of Gastroenterology, vol. 20, no. 43, pp. 1622716235, 2014.

57. Aroor A, J. Habibi, D. A. Ford et al., "Dipeptidyl peptidase-4 inhibition ameliorates western diet-induced hepatic steatosis and insulin resistance through hepatic lipid remodeling and modulation of hepatic mitochondrial function," Diabetes, vol. 64, no. 6, pp. 1988-2001, 2015.

58. Macauley M, K. G. Hollingsworth, F. E. Smith et al., "Effect of vildagliptin on hepatic steatosis," The Journal of Clinical Endocrinology \& Metabolism, vol. 100, no. 4, pp. 1578-1585, 2015.

59. Xiaoqing Ma, Wenhua Du, Shanshan Shao, Chunxiao Yu, Lingyan Zhou, Fei Jing, "Vildagliptin Can Alleviate Endoplasmic Reticulum Stress in the Liver Induced by a High Fat Diet", BioMed Research International, vol. 2018
60. Lee A, Scapa E, Cohen D, and Glimcher L, "Regulation of hepatic lipogenesis by the transcription factor XBP1," Science, vol. 320, no. 5882, pp. 1492-1496, 2008.

61. Wang X, Hausding M, Weng SY, Kim YO, Steven S, Klein T. Gliptins Suppress Inflammatory Macrophage Activation to Mitigate Inflammation, Fibrosis, Oxidative Stress, and Vascular Dysfunction in Models of Nonalcoholic Steatohepatitis and Liver Fibrosis. Antioxidants Redox Signal [Internet]. 2018 Jan 10 ;28(2):87$109 ; 2020$.

62. Joy TR, McKenzie CA, Tirona RG, Summers K, Seney S, Chakrabarti S, Sitagliptin in patients with non-alcoholic steatohepatitis: A randomized, placebo-controlled trial. World $J$ Gastroenterol;23(1):141-50;2017. 